\title{
Correction
}

\section{Correction: Reardon et al., An Allometric Analysis of Sex and Sex Chromosome Dosage Effects on Subcortical Anatomy in Humans}

In the article "An Allometric Analysis of Sex and Sex Chromosome Dosage Effects on Subcortical Anatomy in Humans" by Paul Kirkpatrick Reardon, Liv Clasen, Jay N. Giedd, Jonathan Blumenthal, Jason P. Lerch, M. Mallar Chakravarty, and Armin Raznahan, which appeared on pages 2438-2448 of the February 24, 2016 issue, there were small typographical errors that the authors would like to correct to aid in the reading and interpretation of the article. The errors do not affect any of the conclusions or interpretations in our paper. The list of text corrections as highlighted in bold below which have also been corrected in the online PDF version.

\section{Author line:}

Paul Kirkpatrick Reardon, Liv S. Clasen, Jay N. Giedd, Jonathan D. Blumenthal, Jason P. Lerch, M. Mallar Chakravarty, Armin Raznahan.

\begin{abstract}
On page $2438,1^{\text {st }}$ and $7^{\text {th }}$ sentence corrected. ... Structural neuroimaging of humans with typical and atypical sex-chromosome complements has established the marked influence of sex and sex chromosome dosage on total brain volume (TBV), and identified potential cortical substrates for the psychiatric phenotypes associated with sex-chromosome aneuploidy (SCA). Here, in a cohort of 354 humans with varying karyotypes (XX, XY, XXX, XXY, XYY, XXYY, XXXXY), we investigate sex and SCA effects on subcortical size and shape-focusing on the striatum, pallidum and thalamus. We find large effect-size differences in the volume and shape of all three structures as a function of sex and SCA. We correct for TBV effects with a novel allometric method harnessing normative scaling rules for subcortical size and shape in humans, which we derive here for the first time. We show that all three subcortical volumes scale sub-linearly with TBV amongst healthy humans - mirroring known relationships between subcortical volume and TBV amongst species. Traditional TBV correction methods assume linear scaling and can therefore invert or exaggerate sex and SCA effects on subcortical anatomy. Allometric analysis restricts sex differences to (i) greater pallidal volume (PV) in males, and (ii) relative caudate head expansion and ventral striatum contraction in females. Allometric analysis of SCA reveals that supernumerary X- and Y-chromosomes both cause disproportionate reductions in PV, and coordinated deformations of striatothalamic shape. Our study provides a novel understanding of sex and sex-chromosome dosage effects on subcortical organization, using an allometric approach that can be generalized to other basic and clinical structural neuroimaging settings.
\end{abstract}

\section{Materials and Methods}

On page $2441,1^{\text {st }}$ paragraph below Figure 1, on line $4 . \ldots$ To achieve this, we first re-expressed mean vertex-wise surface area (SA) measures in each SCA group as effect-size deviations from SA distributions at homologous vertices in the appropriate gonadally matched control group (i.e., XXX mean against the XX distribution/XXY, XYY, XXYY, and XXXXY means against the XY distribution).

These steps resulted in a matrix of 21156 rows (all subcortical vertices) and 5 columns (effect-size for SA in each SCA group vs. its gonadal control).

This matrix was then submitted to a k-means partitioning algorithm ["kmeans" function in the R language and environment for statistical computing (R Core Team, 2015; Wickham, 2009); with iter.max and nstart set at 100], and an optimal four-cluster solution was indicated by visual inspection of a scree-plot charting reduction in the mean within-partition sums of squares against increasing partition number.

\section{Results}

On page $2444,2^{\text {nd }}$ column, below Figure 4, line $6 . \ldots$ Comparison of the observed effects of SCA on regional subcortical area with regional areal changes predicted from normative allometry revealed that only a subset of regional SCA effects on subcortical shape reflected unexpected deviations from what would be predicted by normative allometry alone: namely, XXX, XXY, XXYY, and XXXXY effects on the area of blue and red domains within the striatum and thalamus.

\section{Discussion}

On page $2445,2^{\text {nd }}$ column, below subhead Allometry of subcortical volumes, line $7 . \ldots$ In contrast, analysis of our data using two classical "corrections" for TBV effects, which both fail to account for nonlinear relationships between subcortical volumes and TBV, concludes that male sex imparts smaller SV, TV, and PV (normalization by TBV), or tends toward smaller SV and TV, alongside significantly larger PV (covarying for TBV).

Our analyses using these classical corrections recapitulate previous findings of normative sex-differences in subcortical volumes using normalization by, or covariation for TBV (Ahsan et al., 2007; Rijpkema et al., 2012).

On page $2446,2^{\text {nd }}$ column, below subhead Allometry of subcortical shape, 3rd paragraph, line 1 . ... Our analyses of normative subcortical allometry hint that sex could potentially influence normative relationships between TBV and subcortical shape; e.g., cyan 
and red domains of the thalamus show allometric scaling with thalamic size in females (hyper- and hypoallometric, respectively) but isometric scaling in males.

On page 2447, $1^{\text {st }}$ column, line $4 . \ldots$ Given the divergent gene content of human X-and Y-chromosomes, convergent supernumerary X-and Y-chromosome effects on subcortical anatomy could potentially reflect: (1) increased dose of homologous X-Y gene pairs (Otto et al., 2011; Bellott et al., 2014), or (2) increased nuclear chromatin dosage.

\section{References}

R Core Team (2015). R: A language and environment for statistical computing. R Foundation for Statistical Computing, Vienna, Austria. URL https://www.R-project.org/

Wickham, H. ggplot2: Elegant Graphics for Data Analysis. Springer-Verlag New York, 2009.

DOI: 10.1523/JNEUROSCI.1171-16.2016 